\title{
Increasing levels of Parasutterella in the gut microbiome correlate with improving low- density lipoprotein levels in healthy adults consuming resistant potato starch during a randomised trial
}

\author{
Jason R. Bush ${ }^{1,2^{*}}$ id and Michelle J. Alfa ${ }^{3}$
}

\begin{abstract}
Background: Prebiotics, defined as a substrate that is selectively utilized by host microorganisms conferring a health benefit, present a potential option to optimize gut microbiome health. Elucidating the relationship between specific intestinal bacteria, prebiotic intake, and the health of the host remains a primary microbiome research goal.

Objective: To assess the correlations between gut microbiota, serum health parameters, and prebiotic consumption in healthy adults.

Methods: We performed ad hoc exploratory analysis of changes in abundance of genera in the gut microbiome of 75 participants from a randomized, placebo-controlled clinical trial that evaluated the effects of resistant potato starch (RPS; MSPrebiotic ${ }^{\oplus}, N=38$ ) intervention versus a fully digestible placebo ( $\left.N=37\right)$ for which primary and secondary outcomes have previously been published. Pearson correlation analysis was used to identify relationships between health parameters (ie. blood glucose and lipids) and populations of gut bacteria.

Results: Abundance of Parasutterella (phylum Proteobacteria) tended to increase in the gut microbiome of individuals consuming RPS and those increases in Parasutterella were correlated with reductions in low-density lipoprotein (LDL) levels in participants consuming RPS but not placebo. Segregating RPS-consuming individuals whose LDL levels decreased (ie "Responders") from those who did not (ie. "Non-Responders") revealed that LDL Responders had significantly higher levels of Parasutterella both at baseline and after 12 weeks of consuming RPS.
\end{abstract}

Conclusion: Our analyses suggest that RPS may help improve LDL levels depending upon the levels of Parasutterella in an individual's gut microbiome.

* Correspondence: jbush@MSPrebiotics.com

'MSP Starch Products Inc., Carberry, MB, Canada

2Department of Biology, Brandon University, Brandon, MB, Canada

Full list of author information is available at the end of the article

(c) The Author(s). 2020 Open Access This article is licensed under a Creative Commons Attribution 4.0 International License, which permits use, sharing, adaptation, distribution and reproduction in any medium or format, as long as you give appropriate credit to the original author(s) and the source, provide a link to the Creative Commons licence, and indicate if changes were made. The images or other third party material in this article are included in the article's Creative Commons licence, unless indicated otherwise in a credit line to the material. If material is not included in the article's Creative Commons licence and your intended use is not permitted by statutory regulation or exceeds the permitted use, you will need to obtain permission directly from the copyright holder. To view a copy of this licence, visit http://creativecommons.org/licenses/by/4.0/. The Creative Commons Public Domain Dedication waiver (http://creativecommons.org/publicdomain/zero/1.0/) applies to the data made available in this article, unless otherwise stated in a credit line to the data. 
(Continued from previous page)

Trial registration: This study protocol was reviewed and approved by Health Canada (Submission \#188517; "Notice of Authorization" dated 06/05/13) and registered as NCT01977183 (10/11/13) listed on NIH website: ClinicalTrials.gov.

Data generated in this study have been submitted to NCBI (http://www.ncbi.nlm.nih.gov/bioproject/381931).

Funding: MSP Starch Products Inc.

Keywords: Parasutterella, Proteobacteria, Potato, Resistant starch, LDL, Cholesterol

\section{Background}

The ecosystem of microbes in the human intestines, often referred to as the gut microbiome, influences a wide range of physiological processes and methods to manipulate these connections are actively being investigated [1]. Prebiotics, defined as "a substrate that is selectively utilized by host microorganisms conferring a health benefit", stimulate the growth of certain populations of beneficial microbes and therefore offer a strategy to favorably alter the gut microbiome [2]. Prebiotic consumption can positively affect the physiology of the host as well as the microbiome [3, 4], motivating further investigation into the potential health benefits of prebiotics. While the relationships between specific gut microorganisms, dietary intake, and host health outcomes are broadly applicable in principle, dietary interventions to promote host health outcomes may have varying effects depending on the baseline composition of the host's gut microbiome [5]. This reflects both the promise and the challenge of capitalizing on personalized nutrition.

We previously described a clinical trial examining the effects of the MSPrebiotic ${ }^{\circledR}$ (also marketed as the ingredient Solnul ${ }^{\mathrm{TM}}$ ) resistant potato starch (RPS) on the microbiome and various health parameters in people 30-50 years old and those 70 years of age or older [3, 4]. We demonstrated that RPS led to significant increases in Bifidobacterium, reductions in Escherichia, enhanced butyrate production [4], and improvements in blood glucose, insulin, and insulin resistance [3] that were correlated with reductions in the abundance of Sporacetigenium [6].

The connections between starch-fermenting Bifidobacterium, butyrate-producing members of the phylum Firmicutes, and health outcomes have been well-documented [7] but little research has been done to establish connections between other, more obscure bacteria in the gut microbiome. Furthermore, the role of Proteobacteria in the gut microbiome remains ambiguous, with evidence supporting both healthy [8] and pathogenic relationships [9]. Here, we report that levels of Parasutterella (phylum Proteobacteria), a core member of the gut microbiota [10], were increased in a subset of people consuming RPS. The objective of this study was to explore connections between Parasutterella and various metabolic markers in RPS- consuming individuals to determine the relevance of this gut microbiome alteration.

\section{Methods \\ Clinical study, sample collection, and processing}

The primary and secondary endpoints of this prospective, randomized, blinded, placebo-controlled study have been previously described in detail [3, 4]. Study design, enrollment, analysis, and interpretation follow CONSORT guidelines, and a full study protocol is available upon request. Enrollment began in September 2013 and was completed in May 2015. Adults (aged 30-50 years or aged 70 years or older; 75 analyzed; Fig. 1) were recruited in Winnipeg, MB, Canada. Participants (or an authorized third party) provided written informed consent in accordance with the Declaration of Helsinki and in compliance with the University of Manitoba guidelines. Participants were advised that they could withdraw from the study at any time without negative consequences. Exclusion criteria included: Pregnancy, diagnosis with Crohn's disease or other inflammatory bowel disease, systemic lupus erythematosus, prediabetes or diabetes, thyroid disease, renal disease, hepatic disease, dysphagic individuals or those who previously had gastrointestinal surgery (intestinal resection, gastric bypass or colorectal surgery), those on cancer chemotherapy, consuming probiotics or fermented foods (ie. yogurt), on antibiotics at recruitment or within the 5 previous weeks, subjects using additional fiber supplements, and individuals on digestants, emetics, anti-emetics, medications for acid peptic disorders, or antacids. Participants consumed $30 \mathrm{~g}$ of placebo (digestible corn starch; Amioca TF, Ingredion, Brampton, ON, Canada) daily for 2 weeks before randomization to placebo $(N=37)$ or resistant potato starch (RPS as MSPrebiotic ${ }^{\circ}$; MSP Starch Products Inc., Carberry, MB, Canada; $N=38$ ) arms (Fig. 1). RPS and placebo products were comparable in appearance and organoleptic properties. Participants were assigned to placebo or study product by the study coordinator based on a list of computer-generated randomized numbers, and then consumed $30 \mathrm{~g}$ of placebo or RPS daily for 12 weeks (14 weeks total). Stool and fasting blood samples were collected at baseline and 14 weeks. Blood glucose and lipid (total cholesterol, triglycerides, lowdensity lipoprotein, high-density lipoprotein) levels were determined by Diagnostic Services Manitoba (Winnipeg, $\mathrm{MB}$ ) and insulin levels by LipoScience Inc. (Raleigh, NC). 


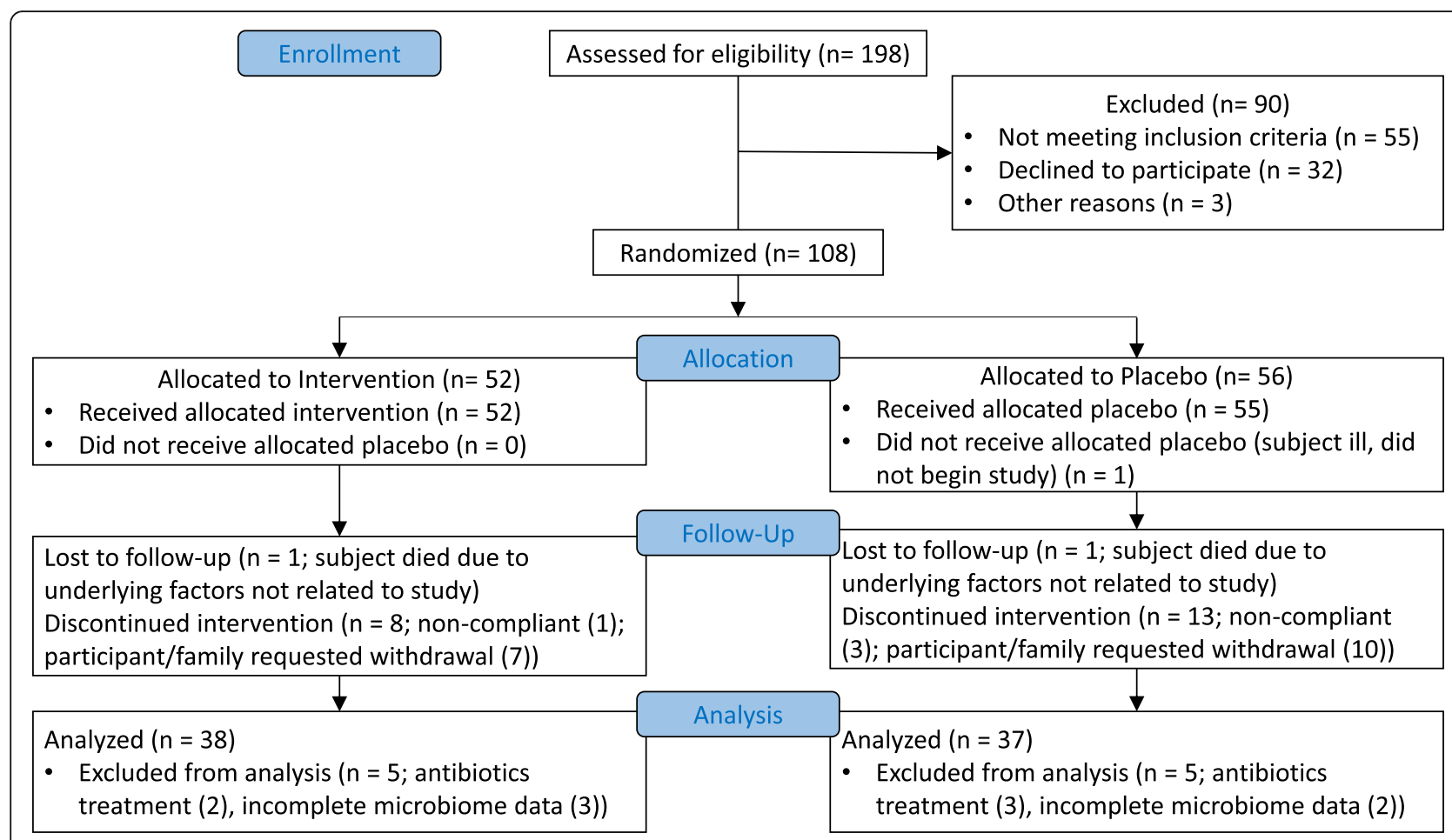

Fig. 1 CONSORT Flow Diagram. Number of participants analyzed; RPS $n=38$, placebo $n=37$, unless otherwise specified

Gut microbiome analysis was performed by $16 \mathrm{~S}$ rRNA sequencing on the Illumina MiSeq platform and alignment as previously described $[4,11,12]$. The data from all participants (regardless of age) was pooled for this analysis. Care providers, trial participants, laboratory testing personnel, and data analysts were blinded to which arm participants were assigned.

\section{Amino acid analysis}

Samples of RPS (MSP Starch Products Inc., Carberry, $\mathrm{MB}$, Canada) and green banana starch (Natural Evolution, Walkamin, QLD, Australia) were sent to Bureau Veritas Laboratories (Mississauga, ON, Canada) and amino acid contents were determined using AOAC 982.30 methods.

\section{Statistical analysis}

Baseline values were subtracted from week 14 values and expressed as a change in percent (blood lipid, glucose, and insulin levels) or a change in relative abundance (bacteria) for each participant. Pearson's correlation coefficients $(r)$ for changes in Parasutterella and changes in blood chemistry were calculated and $p$ values determined using Excel (Microsoft, Redmond, WA), as were Student's one-way t-Test calculations. We employed the Benjamini-Hochberg procedure [13] at a false discovery rate (FDR; $q$ ) of 0.1 to control for multiple testing during correlation analysis. The critical values for each parameter were generated by dividing the $p$ value rank $(i)$ by the total number of parameters analyzed $(m)$, then multiplying this quotient by the $\operatorname{FDR}(q)$. Microbiome variability was expressed as the standard error of the mean (SEM) while blood chemistry variability was expressed as the standard deviation (SD), with $p<0.05$ considered statistically significant.

\section{Results}

RPS consumption led to increases in some genera of bacteria and decreases in others [4, 6] (Fig. 2) while changes in response to placebo were minor (Fig. 3). The effects on Bifidobacterium have previously been described [4] and here we find that RPS significantly increased Clostridium $(p=0.020716)$ and decreased Blautia $(p<0.000001)$, but had no significant effects on Staphylococcus ( $p=$ 0.323284) or Faecalbacterium ( $p=0.094695)$. We previously reported that RPS consumption led to reduced Escherichia coli levels [4] but Parasutterella was the only genus belonging to phylum Proteobacteria that increased in those consuming RPS (Fig. 2). This two-fold increase trended towards significance in RPS consumers (Fig. 4a, $p$ $=0.0711$ ) but placebo consumption had no effect on Parasutterella levels (Fig. 4a, $p=0.291$ ).

We previously demonstrated that decreases in Sporacetigenium were correlated with improvements in blood glucose and insulin in people consuming RPS [6], so we investigated whether increases in Parasutterella in response 


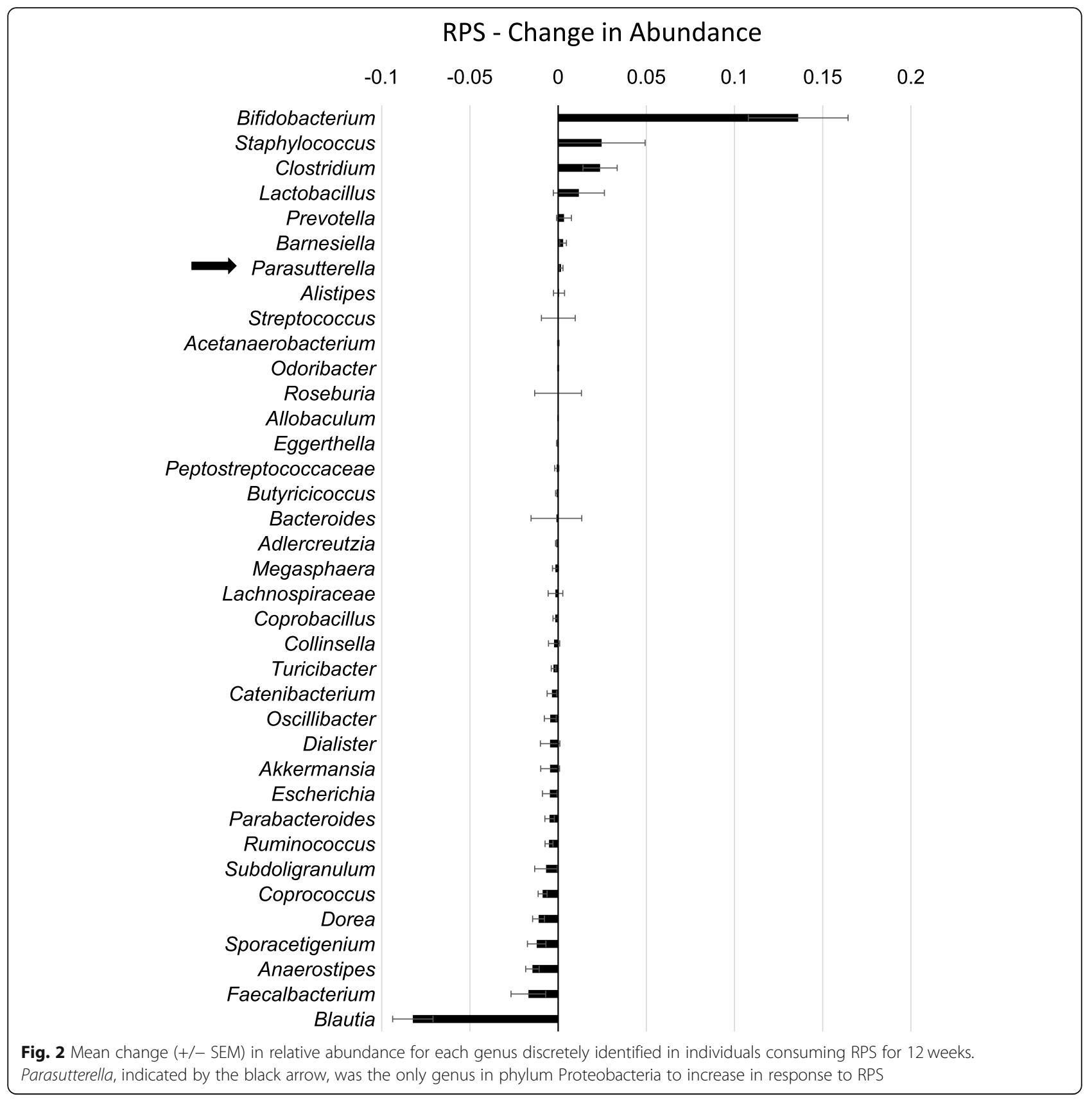

to RPS consumption were correlated with markers of cardiovascular and/or metabolic health. Pearson correlation coefficients $(r)$ and $p$ values were calculated for changes in Parasutterella and changes in total cholesterol, triglycerides, low-density lipoprotein (LDL), high-density lipoprotein (HDL), blood glucose, and insulin levels. To control for multiple testing, the $p$ values were then compared for significance using the Benjamini-Hochberg method (Table 1). Changes in Parasutterella were significantly inversely correlated with changes in LDL levels (i.e. as Parasutterella increased, LDL levels decreased) in individuals consuming RPS ( $r=-0.400461 ; p=0.01284)$ but not with the other parameters. Notably, changes in Parasutterella were not significantly correlated with LDL levels in the placebo group $(r=0.230647 ; p=0.1697)$.

Despite the correlation between changes in Parasutterella and changes in LDL levels, RPS consumption did not lead to overall changes in LDL compared to placebo-consuming controls [4]. Parasutterella is found in low abundance in the gut microbiome [10], suggesting that a minimum threshold may be required for RPS-mediated effects on LDL. We therefore evaluated whether baseline Parasutterella levels were higher in RPS consumers who experienced a decrease in LDL levels (Responders) compared to those 


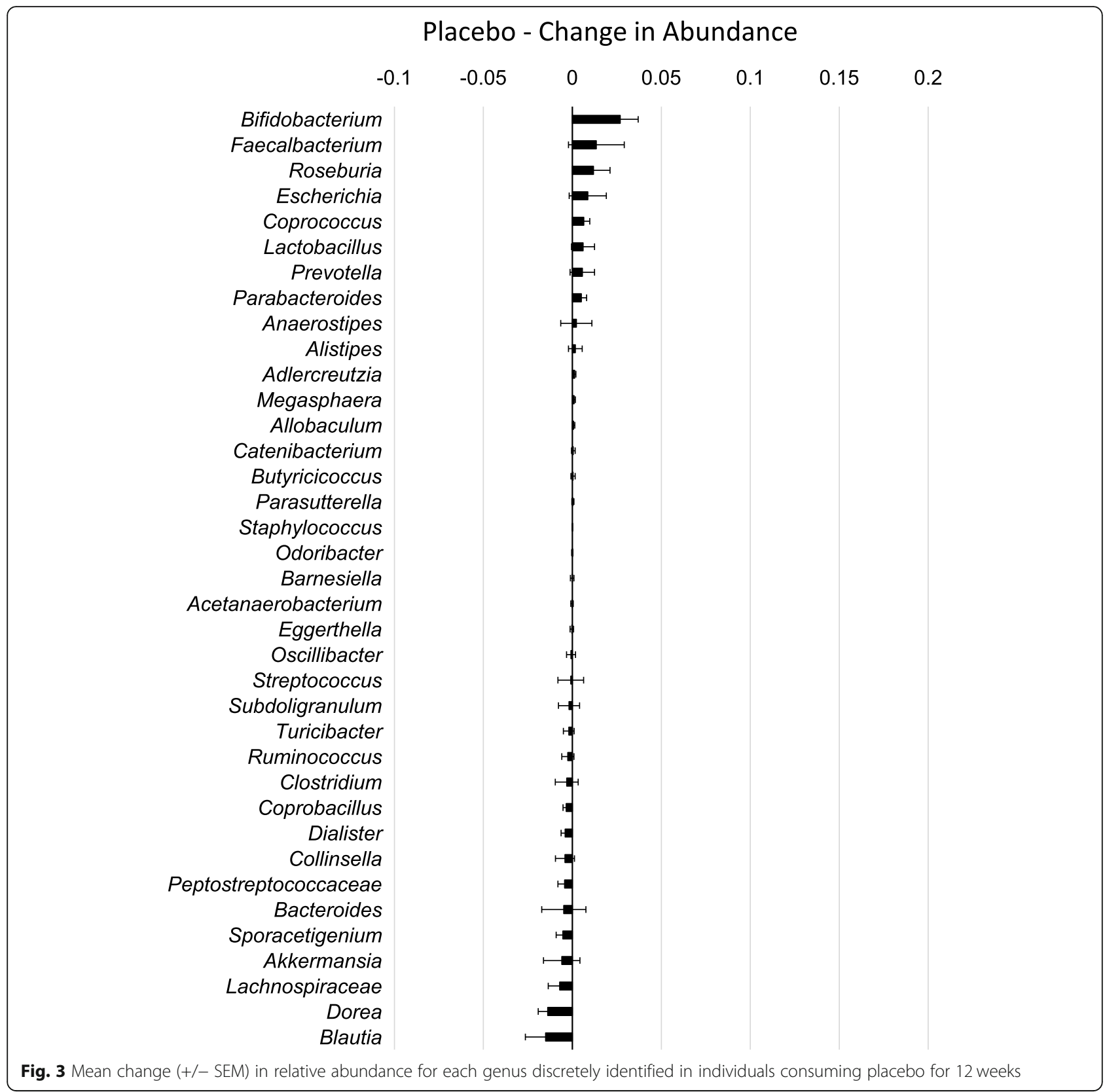

in which LDL levels increased or were unchanged (Non-Responders). Consistent with this hypothesis, we found that baseline Parasutterella levels were significantly more abundant in Responders compared to Non-Responders (Fig. 4b, $p=0.03892$ ). This difference between Responders and Non-Responders was more pronounced at the end of the trial (Fig. 4b, $p=0.04401$ ), although Responders' Week 14 levels were not significantly different from those at baseline $(p=0.15267)$.

It is possible that LDL changes observed are independent of RPS supplementation. For example, Responders may have high baseline LDL levels that decrease over time and Non-Responders may have a low baseline LDL levels that rise over time. To address this possibility, we segregated participants in the Placebo group into Responders (LDL levels decrease) and Non-Responders (LDL levels increase or are unchanged) as described for the RPS group. We found that baseline LDL levels were significantly different between Responders and Nonresponders in the placebo group $(p=0.00245)$ but nearly identical at Week 14 ( $p=0.91978$; Fig. 5a). Thus, the LDL responses in the placebo group were due to differences in baseline levels prior to intervention. Conversely, baseline LDL levels were nearly identical between Responders and Non-Responders in the RPS group ( $p=$ $0.85119)$ but significantly different at Week 14 $(p=$ 


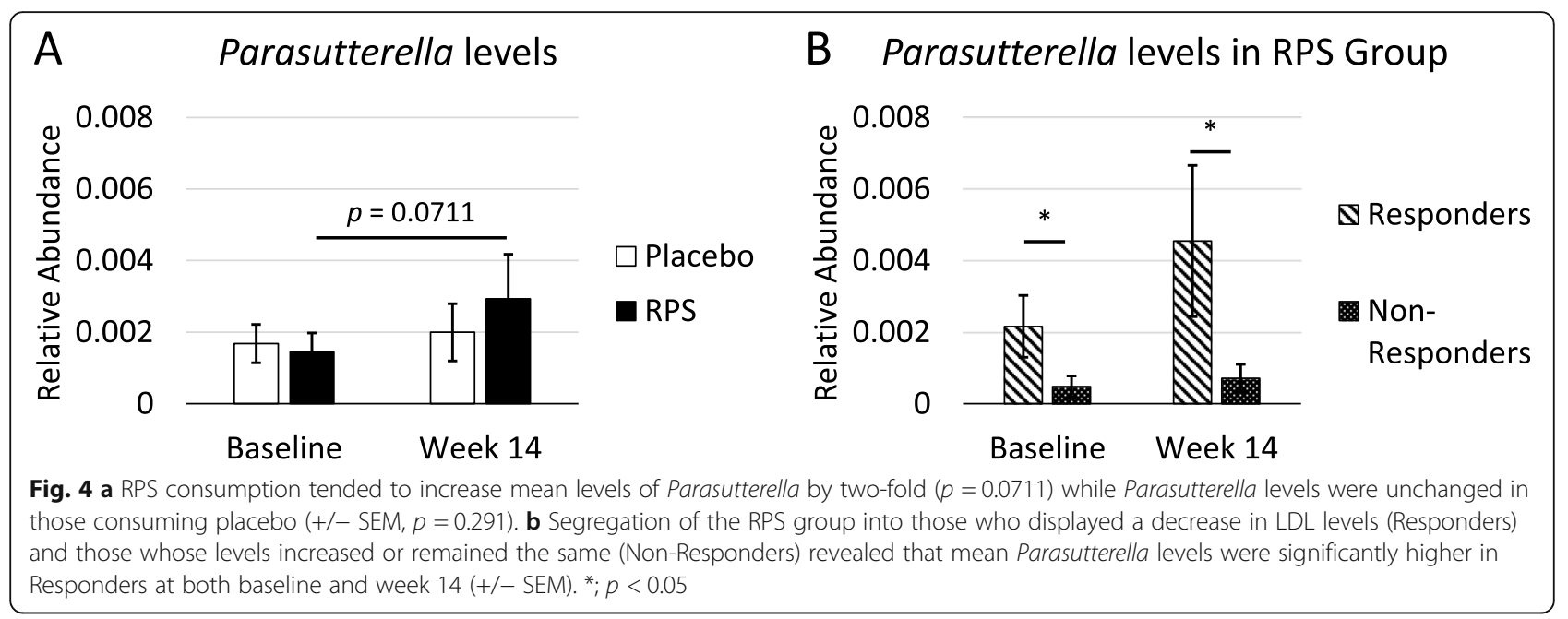

0.00814; Fig. 5b), which indicates the LDL responses in the RPS group are due to the intervention. Taken together, these data suggest that RPS consumption leads to reduced LDL levels in individuals with abovethreshold levels of Parasutterella and that serum LDL response is inversely proportional to Parasutterella changes in the stool.

Characterization of Parasutterella revealed a genomic signature consistent with an inability to ferment starch, and in vitro culture assays demonstrated preferential catabolism of non-essential amino acids and growth on non-essential amino acid substrates such as L-asparagine and L-aspartic acid [10]. Given that potato starches have previously been demonstrated to contain these amino acids [14], we assessed whether RPS similarly contained amino acids that could support the growth of Parasutterella. Unlike green banana starch, RPS contained no amino acids except trace amounts of tryptophan (Table 2). This suggests that increased levels of Parasutterella in the gut microbiome in response to RPS supplementation is not due to L-asparagine and Laspartic acid derived from RPS [10].

\section{Discussion}

While species belonging to the genera Bifidobacterium and Ruminococcus are generally recognized as the only primary degraders of resistant starch [15], consumption of resistant potato starch (RPS) led to significant changes in several different genera, including those belonging to phylum Proteobacteria. Members of the gut microbiome belonging to Proteobacteria are typically considered to be undesirable, and are associated with infectious diarrhea, elevated inflammation, increased permeability of the gut wall and the production of harmful metabolites [16]. Here, we sought to understand subtle changes in non-primary resistant starch degraders and report a correlation between increasing levels of Parasutterella (phylum Proteobacteria), a core member of the gut microbiota [10], and decreasing levels of LDL, an important risk factor for cardiovascular disease. Notably, the linear correlation between increases in Parasutterella and decreases in LDL facilitates integration into gut microbiome testing platforms, such that interventions (such as prebiotic or probiotic supplementation) that increase Parasutterella will be predicted to similarly reduce LDL levels in a proportional manner. While the effect of RPS on LDL

Table 1 Correlations between the change in abundance of Parasutterella and changes in total cholesterol, triglycerides, low-density lipoprotein (LDL), high-density lipoprotein (HDL), blood glucose, and insulin levels in individuals consuming RPS

\begin{tabular}{|c|c|c|c|c|}
\hline Health Parameter & $r$ & $p$ value & Rank (i) & Critical Value $\left([i / m]^{*} q\right)$ \\
\hline LDL & -0.40046151 & 0.01284 & 1 & 0.016667 \\
\hline Blood Glucose & 0.256738899 & 0.119769 & 2 & 0.033333 \\
\hline Cholesterol & -0.18932218 & 0.255775 & 3 & 0.05 \\
\hline Insulin* & 0.130393557 & 0.448782 & 4 & 0.066667 \\
\hline $\mathrm{HDL}$ & -0.06547371 & 0.698229 & 5 & 0.083333 \\
\hline Triglycerides & 0.037206775 & 0.824522 & 6 & 0.1 \\
\hline
\end{tabular}

The Benjamini-Hochberg method controls for false discovery of significant correlations [13]. Results are rank ordered based on $p$ value, and the $p$ value is compared to the critical value $\left([i / m]^{*} q\right.$; FDR $\left.(q)=0.1\right)$ beginning with the lowest ranking parameter (Triglycerides). The first correlation with a $p$ value lower than the critical value (LDL) and any higher-ranking correlations are considered significant (bold). Positive Pearson correlation coefficient ( $r$ ) values indicate positive correlations and negative $r$ values indicate negative correlations. $N=38$ except for Insulin*, where $N=36$ due to missing insulin measurements 


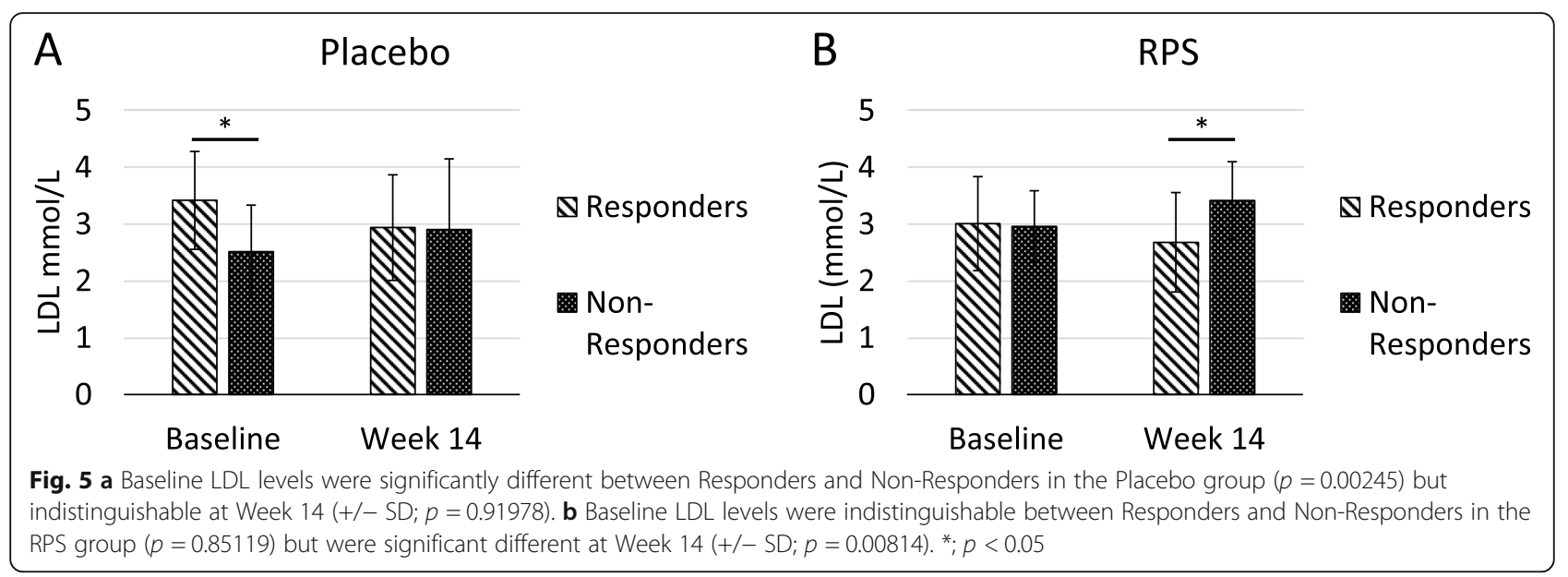

cholesterol was modest (Fig. 5b; mean change - $0.33 \mathrm{mmol} /$ $\mathrm{L}$ in Responders) compared to cholesterol lowering medications [17], RPS supplementation may help reduce LDL levels in combination with other therapies. The evaluation of Parasutterella levels in the gut microbiome to predict a person's response to RPS is consistent with a personalized approach to medicine and speaks to growing appreciation

Table 2 Amino acid abundance in resistant starch from potato and green banana sources

\begin{tabular}{lll}
\hline Amino Acid & Resistant Potato Starch & Green Banana Starch \\
\hline Alanine & ND & 0.16 \\
Arginine & ND & 0.21 \\
Aspartic Acid & ND & 0.59 \\
Cystine & ND & ND \\
Glutamic Acid & ND & 0.56 \\
Glycine & ND & 0.14 \\
Histidine & ND & 0.13 \\
Isoleucine & ND & 0.10 \\
Leucine & ND & 0.19 \\
Lysine & ND & 0.14 \\
Methionine & ND & 0.04 \\
Phenylalanine & ND & 0.13 \\
Proline & ND & 0.15 \\
Serine & ND & 0.14 \\
Taurine & ND & 0.05 \\
Threonine & ND & 0.11 \\
Tryptophan & 0.05 & 0.08 \\
Tyrosine & ND & 0.08 \\
Valine & ND & 0.14 \\
Total & $\mathbf{0 . 0 5}$ & $\mathbf{3 . 1 4}$ \\
\hline Amino aids & &
\end{tabular}

Amino acids measured using the AOAC 982.30 methodology. All values are reported as $\mathrm{g} / 100 \mathrm{~g}$. The reportable detection limit for each amino acid is 0.01 g/100 g. ND Not detected for the role that differences in gut microbiome composition play in shaping human health.

Parasutterella is an anaerobic, asaccharolytic Gramnegative, non-spore-forming coccobacillus, that was originally described based on a strain isolated from a fecal sample from a healthy Japanese male [18]. Deep sea water (DSW) is one of several dietary supplements that tend to increase Parasutterella levels [1922]. Using a diet-induced hamster model of hypercholesterolemia, Lin and colleagues demonstrated that DSW also led to significant reductions in triglycerides, LDL, and total cholesterol, although Bacteroidetes was the only bacterial population significantly correlated with serum cholesterol and LDL in response to the high cholesterol diet [20]. Increases in Parasutterella in response to GOS supplementation in mice were associated with significant reductions in triglycerides but not LDL levels [23]. Parasutterella levels were inversely correlated with fat consumption but not total energy intake in obese people [24].

Introduction of Parasutterella into normal mice led to reductions in cecal levels of cholic acid, taurocholic acid, taurodeoxycholic acid, 7-ketodeoxycholic acid (or isomers), and glycolithocholic acid [10]. Additionally, there were concomitant increases in taurine and changes in bile acid metabolism that were consistent with bacteria-mediated deconjugation of primary bile acids [10]. Furthermore, the same study demonstrated changes in farnesoid X receptor (FXR)-dependent gene expression, including increases in Cyp7a1, suggesting enhanced bile acid synthesis [10]. Mushroom polysaccharide supplementation of a high fat diet led to similar changes in gene expression, along with increases in Parasutterella that were correlated with reductions in serum lipids [25]. While total cholesterol levels were decreased, although not significantly, by the introduction of Parasutterella, LDL levels were not measured [10], suggesting that the mechanisms documented in mice could be acting similarly in humans consuming RPS, specifically 
those with above-threshold levels of Parasutterella. Furthermore, although stable colonization of Parasutterella in the gastrointestinal tract occurred rapidly in mice [10], it is possible that the mean changes in bile acid abundance, FXR-dependent gene expression, and the effects on cholesterol are driven by mice in which higher levels of Parasutterella engrafted to the gut microbiome, consistent with the data presented here for humans.

Despite potatoes having been identified as dietary sources of asparagine and aspartic acid [14], our analysis reveals that all amino acids, except for a trace amount of tryptophan, are absent in RPS. Thus, it is unclear from our data how RPS supports the growth of Parasutterella. It is intriguing to note that Parasutterella was identified as part of a co-abundance response group that increased in response to chemically modified resistant starches (Type 4) in people [26]. This suggests that resistant starch may generally support the growth of Parasutterella, through cross-feeding or some other indirect mechanism(s). Indirect growth via complex ecological interactions could explain why RPS consumption stimulated growth of Parasutterella in some people and not others. Furthermore, Fig. 2 demonstrates that there are a number of significant microbial shifts within the gut microbiome that occur in humans consuming RPS (e.g. Bifidobacteria and Clostridium increase while Blautia decrease). This suggests there may be a "co-abundance" type response in humans related to RPS consumption, though the interactions between Parasutterella and these genera remain to be elucidated. This supports Marchesi and others' statement [27]: "However, as we learn more about the ecology of the gut microbiota it is becoming clear that the prebiotic concept has tapped into the underlying fabric of the gut microbiota as a primarily saccharolytic and fermentative microbes community evolved to work in partnership with its host's digestive system to derive energy and carbon from complex plant polysaccharides which would otherwise be lost in faeces."

Limitations of our study include the large dose (30 g/day), the relatively small study population, and the use of age brackets within that population. Furthermore, statistical analysis of Parasutterella levels was hampered by the low relative abundance of this genus (mean levels $<0.4 \%$ ). Future studies examining the response of Parasutterella to lower doses of RPS in a larger, general population are warranted.

\section{Conclusions}

We propose that the effect of Parasutterella on the host's physiology is dependent upon a variety of factors, including prebiotic consumption, the baseline level of this organism and the co-abundance response of the host's gut microbiome. Further research is required to identify these factors and the mechanisms by which they interact to influence cholesterol homeostasis.

\section{Abbreviations}

DSW: Deep sea water; FDR: False discovery rate; FXR: Farnesoid X receptor; HDL: High density lipoprotein; LDL: Low density lipoprotein; ND: Not detected; RPS: Resistant potato starch; SD: Standard deviation; SEM: Standard error of the mean

\section{Acknowledgements}

We thank the clinical trial participants, as well as David Strang, Michael Kaan, Daryl Dyck, Jo-Ann Lapointe-McKenzie, Jean Helps, Nila McFarlane, Betty Smith (Deer Lodge, Winnipeg); Brendan Dufault, Lisa Lix (University of Manitoba); Jessica Forbes, Morag Graham, Gary Van Domselaar, Vanessa Laminman (National Microbiology Laboratory, Public Health Agency of Canada); Paramjit Tappia, Nancy Olson, Pat DeGagne, David Bray, Brenda-Lee Murray (St. Boniface Research Centre); and Brett Hiebert (Asper Clinical Research Institute).

\section{Authors' contributions}

MJA designed the clinical trial and was responsible for overseeing the collection and analysis of blood and stool samples. JRB performed the statistical analysis, generated the figures and tables, and wrote the manuscript. Both authors read and approved the final version of the manuscript.

\section{Funding}

Funding provided by MSP Starch Products Inc., Carberry, MB, Canada, which was the study sponsor.

\section{Availability of data and materials}

Data generated have been submitted to NCBI (http://www.ncbi.nlm.nih.gov/ bioproject/381931).

\section{Ethics approval and consent to participate}

Research and ethics approval and protocol modification approvals were obtained from Health Canada and the University of Manitoba Research Ethics Board before implementation. Health Canada authorized the study (Submission \#188517) on June 5, 2013, which was registered on

ClinicalTrials.gov (October 11, 2013; NCT01977183). Procedures were followed in accordance with Health Canada and University of Manitoba ethical standards. Participant identifiers were treated in confidence and according to the Personal Health Information Act of Manitoba. Study information was locked in a secured area and information sent for statistical analyses had no participant identifiers. The University of Manitoba Office, Research Quality Management unit independently audited the study.

\section{Consent for publication}

Not applicable.

\section{Competing interests}

Jason Bush is employed by and Michelle Alfa provides consulting services for MSP Starch Products Inc., Carberry, MB, Canada, who manufacture MSPrebiotic ${ }^{\circledast}$ resistant potato starch.

\section{Author details}

${ }^{1}$ MSP Starch Products Inc., Carberry, MB, Canada. ${ }^{2}$ Department of Biology, Brandon University, Brandon, MB, Canada. ${ }^{3}$ Department of Medical Microbiology, University of Manitoba, Winnipeg, MB, Canada.

Received: 29 July 2020 Accepted: 15 November 2020 Published online: 11 December 2020

References

1. Cani P. Human gut microbiome: hopes, threats and promises. Gut. 2018; 67(9):1716-25.

2. Gibson G, Hutkins R, Sanders M, Prescott S, Reimer R, Salminen S, Scott K, Stanton C, Swanson K, Cani P, Verbeke K, Reid G. Expert consensus document: the international scientific Association for Probiotics and Prebiotics (ISAPP) consensus statement on the definition and scope of prebiotics. Nat Rev Gastroenterol Hepatol. 2017;14(8):491-502.

3. Alfa MJ, Strang D, Tappia PS, Olson N, DeGagne P, Bray D, Murray B-L, Hiebert B. A Randomized Placebo Controlled Clinical Trial to Determine the Impact of Digestion Resistant Starch MSPrebiotic on Glucose, Insulin, and 
Insulin Resistance in Elderly and Mid-Age Adults. Front Med (Lausanne). 2018;4:260.

4. Alfa MJ, Strang D, Tappia PS, Graham M, Van Domselaar G, Forbes JD, Laminman V, Olson N, DeGagne P, Bray D, Murray B-L, Dufault B, Lix LM. A randomized trial to determine the impact of a digestion resistant. Clin Nutr. 2018:37(3):797-807.

5. Zeevi D, Korem T, Zmora N, Israeli D, Rothschild D, Weinberger A, Ben-Yacov O, Lador D, Avnit-Sagi T, Lotan-Pompan M, Suez J, Mahdi JA, Matot E, Malka G, Kosower N, Rein M, Zilberman-Schapira G, Dohnalová L, Pevsner-Fischer M, Bikovsky R, Halpern Z, Elinav E, Segal E. Personalized nutrition by prediction of glycemic responses. Cell. 2015;163(5):1079-94.

6. Bush JR, Alfa MJ. Decreasing levels of Sporacetigenium correlate with improved diabetic parameters in healthy adults consuming MSPrebiotic ${ }^{\oplus}$ digestion resistant starch. J Aging Res Clin Pract. 2018;7:176-80.

7. De Vuyst L, Leroy F. Cross-feeding between bifidobacteria and butyrateproducing colon bacteria explains bifdobacterial competitiveness, butyrate production, and gas production. Int J Food Microbiol. 2011; 149(1):73-80.

8. Scaldaferri F, Gerardi V, Mangiola F, Lopetuso L, Pizzoferrato M, Petito V, Papa A, Stojanovic J, Poscia A, Cammarota G, Gasbarrini A. Role and mechanisms of action of Escherichia coli Nissle 1917 in the maintenance of remission in ulcerative colitis patients: an update. World J Gastoenterol. 2016;22(24):5505-11.

9. Gomes T, Elias W, Guth SIB, Rodrigues J, Piazza R, Ferreira L, Martinez M. Diarrheagenic Escherichia coli. Braz J Microbiol. 2016;47(Suppl 1):3-30.

10. Ju T, Yoon Kong J, Stothard P, Willing BP. Defining the role of Parasutterella, a previously uncharacterized member of the core gut microbiota. ISME J. 2019;13:1520-34.

11. Schloss PD, Westcott SL, Ryabin T, Hall JR, Hartmann M, Hollister EB, Lesniewski RA, Oakley BB, Parks DH, Robinson CJ, Sahl JW, Stres B, Thallinger GG, van Horn DJ, Weber CF. Introducing mothur: open-source, platformindependent, community-supported software for describing and comparing microbial communities. Appl Environ Microbiol. 2009;75(23):7537-41.

12. Kozich JJ, Westcott SL, Baxter NT, Highlander SK, Schloss PD. Development of a dual-index sequencing strategy and curation pipeline for analyzing amplicon sequence data on the MiSeq Illumina sequencing platform. Appl Environ Microbiol. 2013;79(17):5112-20.

13. Benjamini $Y$, Hochberg $Y$. Controlling the false discovery rate: a practical and powerful approach to multiple testing. J R Stat Soc Series B Stat Methodol. 1995;57(1):289-300.

14. Vivanti V, Finotti E, Friedman M. Level of acrylamide precursors asparagine, fructose, glucose, and sucrose in potatoes sold at retail in Italy and in the United States. J Food Sci. 2006;71(2):C81-5.

15. DeMartino P, Cockburn DW. Resistant starch: impact on the gut microbiome and health. Curr Opin Biotechnol. 2020;61:66-77.

16. Oliphant $\mathrm{K}$, Allen-Vercoe E. Macronutrient metabolism by the human gut microbiome: major fermentation by-products and their impact on host health. Microbiome. 2019;7(1):91.

17. Jones PH, Davidson MH, Stein EA, Bays HE, McKenney JM, Miller E, Cain VA, Blasetto JW, STELLAR Study Group. Comparison of the efficacy and safety of Rosuvastatin versus atorvastatin, simvastatin, and pravastatin across doses (STELLAR* trial). Am J Cardiol. 2003;92(2):152-60.

18. Nagai F, Morotomi M, Sakon H, Tanaka R. Parasutterella excrementihominis gen. Nov., sp. nov., a member of the family Alcaligenaceae isolated from human faeces. Int J Syst Evol Microbiol. 2009:59(Pt 7):1793-7.

19. Chen Y, Xiao S, Gong Z, Zhu X, Yang Q, Li Y, Gao S, Dong Y, Shi Z, Wang Y, Weng X, Li Q, Cai W, Qiang W. Wuji Wan formula ameliorates diarrhea and disordered colonic motility in post-inflammation irritable bowel syndrome rats by modulating the gut microbiota. Front Microbiol. 2017;8:2307.

20. Lin C, Chen Y, Tsai T, Pan T. Effects of deep sea water and lactobacillus paracasei subsp. paracasei NTU 101 on hypercholesterolemia hamsters gut microbiota. Appl Microbiol Biotechnol. 2017;101(1):321-9.

21. Xie M, Chen G, Wan P, Dai Z, Hu B, Chen L, Ou S, Zeng X, Sun Y. Modulating effects of Dicaffeoylquinic acids from llex kudingcha on intestinal microecology in vitro. J Agric Food Chem. 2017;65(47):10185-96.

22. Sun Y, Chen S, Wei R, Xie X, Wang C, Fan S, Zhang X, Su J, Liu J, Jia W, Wang $X$. Metabolome and gut microbiota variation with long-term intake of Panax ginseng extracts on rats. Food Funct. 2018;9(6):3547-56.

23. Cheng W, Lu J, Lin W, Wei X, Li H, Zhao X, Jiang A, Yuan J. Effects of a galacto-oligosaccharide-rich diet on fecal microbiota and metabolite profiles in mice. Food Funct. 2018;9(3):1612-20.
24. Kreutzer C, Peters S, Schulte D, Fangmann D, Türk K, Wolff S, van Eimeren T, Ahrens M, Beckmann J, Schafmayer C, Becker T, Kerby T, Rohr A, Riedel C, Heinsen F, Degenhardt F, Franke A, Rosenstiel P, Zubek N, Henning C, Freitag-Wolf S, Dempfle A, Psilopanagioti A, Petrou-Papadaki H, Lenk L, Jansen O, Schreiber S, Laudes M. Hypothalamic inflammation in human obesity is mediated by environmental and genetic factors. Diabetes. 2017; 66(9):2407-15.

25. Li L, Guo W-L, Zhang W, Xu J-X, Qian M, Bai W-D, Zhang Y-Y, Rao P-F, Ni L, Lv X-C. Grifola Frondosa polysaccharides ameliorate lipid metabolic disorders and gut microbiota Dysbiosis in high-fat diet fed rats. Food Funct. 2019;10(5):2560-72.

26. Deehan EC, Yang C, Perez-Munoz ME, Nguyen NK, Cheng CC, Triador L, Zhang Z, Bakal JA, Walter J. Precision microbiome modulation with discrete dietary Fiber structures directs short-chain fatty acid production. Cell Host Microbe. 2020;27(3):389-404.

27. Marchesi JR, Adams DH, Fava F, Hermes GD, Hirschfield GM, Hold G, Quraishi MN, Kinross J, Smidt H, Tuohy KM, Thomas LV, Zoetendal EG, Hart A. The gut microbiota and host health: a new clinical frontier. Gut. 2016;65: $330-9$.

\section{Publisher's Note}

Springer Nature remains neutral with regard to jurisdictional claims in published maps and institutional affiliations.

\section{Ready to submit your research? Choose BMC and benefit from:}

- fast, convenient online submission

- thorough peer review by experienced researchers in your field

- rapid publication on acceptance

- support for research data, including large and complex data types

- gold Open Access which fosters wider collaboration and increased citations

- maximum visibility for your research: over $100 \mathrm{M}$ website views per year

At BMC, research is always in progress.

Learn more biomedcentral.com/submissions 